\title{
Aggregation Problem in Multi-Regional Input-Output Models*
}

\author{
TAKAO FUKUCHI
}

Graduate School of Business Administration, Asahi University

\begin{abstract}
When a multi - regional and multi - asectoral economy exists, there are many input - output tables at different levels of aggregation. A nm-dimension table for $\mathrm{n}$ - regions and $\mathrm{m}$ - sectors, a $\mathrm{n}$ - regions table, a $\mathrm{m}$ - sectors table and finally a one - region and one - sector (or a multiplier). This paper discusses how these tables can be consistent each other and what are the consistence conditions when final demand changes. First paper clarifies the conditions of consistent aggregation when bottom - up approach is adopted, and shows the prediction errors in traditional calculation based on a numerical example. Then it discusses a decision problem in top - down approach, and finally the roles of technological difference and hysteresis in consistency approach.
\end{abstract}

\section{Introduction}

In this paper I discuss the relationships of parameters of input-output tables at different levels of aggregation. There are an mn-dimension national table with $\mathrm{n}$-regions and $\mathrm{m}$ sectors, a sectoral table with $\mathrm{m}$-sectors, a regional table with $\mathrm{n}$-regions. At the final stage of aggregation, these tables can be aggregated into formulae stating that the total output is a product of total final demand with a single coefficient. Sometimes they are constructed independently from different data sources. So we face with many problems. I classify the relevant problems into two categories : one of theoretical nature and other of statistical nature. The former asks the question, which table represents the actual tendency more accurately? The latter question asks what are the relations between different parameters of different tables?

This paper originates from my actual experiences in a technical cooperation project by JICA to the Indonesian government. At the first stage, the team utilized the 1993 sectoral

*) This paper is an extension of my report with the same title at Japan Regional Science Annual Meeting of 1997. I thank to helpful comments by Prof. Yoshio Kimura, Takeo Ihara and Geoffrey J. D. Hewings. I greatly appreciate the suggestions by Prof. Yoshio Kimura to refine the proofs of Theorem 1-2. I am responsible for any remaining errors. 
table with 28 sectors, and projected the Indonesian economy in coming twenty-five years as a solution of linear programming-type model with capital, skilled labor and foreign currency constraints. As the interregional issues are very important, the team is preparing a full-scale national tables with 28 sectors and 5 regions. Out of four types of interregional input-output tables (Isard, Riefeler-Tiebout, Chenery-Moses, Leontief-Strout ; Hewings-Jensen (1986), p. 303), the aggregation issue is relevant only for such type model. Other three types of models are compromises between Isard and Chenery-Strout type model. But "the full interregional model proposed by Isard (1951), in which an n-sector r-region economy was portrayed by elements $a_{i j}^{p q}$, the flows of output from sector $\mathrm{i}$ in region $\mathrm{p}$ to sector $\mathrm{j}$ in region q, has rarely been implemented empirically" (Hewings-Jensen (1986), p. 302). The preparation of this national multiregional table requires many different data sources beyond the sectoral table. From now on, the team also wants to construct a linear programming-type model utilizing this national table and discuss the several issues like the social cost of mitigating interregional disparity. So the team faces with two problems above mentioned. I start to discuss the statistical problem, and treat the theoretical question at the later stage.

Because I started from such a practical purpose, my approach differs from the theoretical investigation like Hatanaka (1952) and McManus (1956) consolidation approach to seek a condition by which the hybrid model produces the same output as the original model. I basically follow the direction of research a la Theil (1954, p. 5). He defined the consistency approach to aggregation problem, which postulates the consistency between micro and macro models, and seeks to find out the relations between parameters. Then he assumed a similar model or equation at micro and macro levels, and tried to find out the relations. He called this direction as analogy approach (op cit., p. 6). We engage in a similar analysis but for a different topic. We describe the actual economy by input-output table but at different level of aggregation, and discuss the relations between technical coefficients at different levels.

When there are input-output tables at different levels, each table reasonably describes the actual economy from a different aspect. Then what are the relations between technical coefficients of different tables? There are three different approaches in discussing aggregation problems between models of different levels of aggregation : bottom-up, top-down and consistency approaches. Let us take up the case of consumer's behavior. We have the micro data of income and consumption expenditure of each household based on the household expenditure survey, and also the macro data or the aggregates from these micro data. We are interested in the consumer's behavior at both levels. We specify a similar function by these micro and macro data; the parameters in these models are expected to show some similarities when the relationship between micro and macro data is explicitly known. In the simplest case, consumption expenditure of each household $\left(C_{k}\right)$ is assumed to be a linear function of household income $\left(Y_{k}\right)$. 
Aggregation Problem in Multi-Regional Input-Output Models

$$
C_{k}=a_{k}+b_{k} \cdot Y_{k} \quad(k=1, \cdots, N)
$$

We assume that each micro parameter is not same over households. Let us define the macro consumption expenditure $(C)$ and income $(Y)$ by simple sums.

$$
C=\Sigma C_{k}, \quad Y=\Sigma Y_{k}
$$

We specify a similar linear consumption function as follows :

$$
C=A+B \cdot Y
$$

When (1-3) holds for the given micro data set $\left(C_{k}, Y_{k}\right)(k=1, \cdots, N)$, the macro model (parameters) is (are) consistent with the micro model (parameters). This consistency requires that the macro parameters be expressed as suitable functions of macro data and micro parameters :

$$
\begin{aligned}
& A=F\left(C_{k}, Y_{k}, a_{k}, b_{k}\right) \\
& B=G\left(C_{k}, Y_{k}, a_{k}, b_{k}\right)
\end{aligned}
$$

We can intuitively imagine certain relationships between micro and macro parameters $(A$, $B)$. The forms and nature of these relations (1-4) and (1-5) are called as the aggregation problem in linear model. The simplest guess is that the corresponding macro parameters can be expressed as the simple sum or weighted sum of micro parameters.

$$
A=\Sigma a_{k} \text { and } B=\Sigma b_{k} \cdot Y_{k} / Y
$$

But this intuitive guess does not hold in general. So there are three questions or approaches.

(1) Bottom-up Approach : When micro parameters are given, how we can define the consistent macro parameters? This approach tries to fix the functions (1-4) or (1-5) directly.

(2) Top-down Approach: When macro parameter values are given, what are the consistent values of micro parameters? This approach tries to solve (1-4) and (1-5) inversely, and infer the possible values or the range of values of micro parameters consistent with macro parameters.

(3) Consistency Approach : The third approach seeks the conditions to hold the consistency between two models (parameters).

Such national-regional linkages are important in any type of econometric models. Njikamp-Rietveld-Snickers (1986, pp. 279-283) also discussed the three types of treatments of these interactions as bottom-up, top-down and interactive in econometric models. In this paper, we take up a linear model, i. e., multiregional input-output table at different levels of aggregation, and tries to solve the linear aggregation problem by engaging in these three approaches successively. In section 2 we specify the aggregation problem in input-output model, and discuss the bottom-up approach. Section 3 presents a simple numerical example. Section 4 discusses the prediction error when the consistent coefficient matrix is not utilized. Section 5 discusses the top-down approach. Section 6 compares the optimum solutions of national and sectoral models. Section 7 summarizes the conclusions. 
The main interests are the relations between national, regional, sectoral and macro models, and we focus to these relations all through the paper.

\section{Statistical Problem : Bottom-up Approach}

A national economy with m-sectors and n-regions. $X n m, X m, X n$ and $X a$ stand for the vectors of outputs of national model (mn dimensions, $m$-sectors of 1 st region, of $2 \mathrm{nd}$, of $n$th region), of sectoral model (m dimension), of regional model (n dimension) and of macro model (scalar). Similarly, $Y n m, Y m, Y n$ and $Y a$ show the final demand in four models. The basic models are as follows :

(a) National (Multi-Sector-Multi-Region) Model :

$$
(U n m-A n m) \cdot X n m=Y n m
$$

(b) Multi-Sectoral Model :

$$
(U m-A m) \cdot X m=Y m
$$

(c) Multi-Regional Model :

$$
(U n-A n) \cdot X n=Y n
$$

(d) (One-Sector-One-Region) Macro Model :

$$
(1-A a) \cdot X a=Y a
$$

where $U k$ stands for the unit matrix of $k$-dimension. We denote the inverse matrices as follows :

$$
\begin{gathered}
B n m=(U n m-A n m)^{-1}, B m=(U m-A m)^{-1} \\
B n=(U n-A n)^{-1}, B a=(1-A a)^{-1}
\end{gathered}
$$

Let us define the following adding matrices :

$$
\begin{gathered}
L S=(U m, U m, \ldots, U m)(m, n m)-\text { matrix } \\
L R=(V 1, V 2, \ldots, V m)(n, n m)-\text { matrix }
\end{gathered}
$$

where $V k$ is a matrix obtained from an nxm zero matrix by replacing the $k$-th row with the adding (or sum) vector $\mathrm{Jm}$, a row vector consisting of $\mathrm{m}$ ones.

Now we define the consistency between two models as follows :

Definition : National model and sectoral model are consistent each other

$$
\text { iff } X m=L S \cdot X n m \text { and } Y m=L S \cdot Y n m
$$

Definition : National model and regional model are consistent each other

$$
\text { iff } X n=L R \cdot X n m \text { and } Y n=L R \cdot Y n m
$$

Definition : National model and macro model are consistent each other

$$
\text { iff } X a=J n m \cdot X n m \text { and } Y a=J n m \cdot Y n m
$$

Definition : Sectoral model and macro model are consistent each other

$$
\text { iff } X a=J m \cdot X m \text { and } Y a=J m \cdot Y m
$$

Definition : Regional model and macro model are consistent each 


$$
\text { other iff } X a=J n \cdot X n \text { and } Y a=J n \cdot Y n
$$

Definition : Four models are consistent each other, iff (2-9), (2-10), (2-12) and (2-13) hold. Note that

$$
J m \cdot L S=J n \cdot L R=J n m
$$

So (2-11) results from (2-9), (2-10), (2-12) and (2-13).

We are interested in the consistency between the national model and the regional model, postulating (2-11).

National (Sector-Region) Model :

$$
(U n m-A n m) \cdot X n m=Y n m
$$

can be rewritten as :

$$
(L R) \cdot(U n m-A n m) \cdot X n m=L R \cdot Y n m=Y n
$$

Regional Model can be rewritten as :

$$
(U n-A n) \cdot L R \cdot X n m=Y n
$$

Therefore, the consistency condition is

$$
((L R) \cdot(U n m-A n m)-(U n-A n) \cdot L R) \cdot X n m=0
$$

This reduces to

$$
(L R \cdot A n m-A n \cdot L R) \cdot X n m=0
$$

Clearly, this is the necessary and sufficient condition for consistency between national model and regional model. To secure (2-18), An must be a special function of parameters and variables at national level.

$$
A n=F(A n m, X n m)
$$

$Y n m$ can be determined from $A n m$ and $X n m$. The bottom-up approach is to find out a suitable form of (2-19), and express $A n$ as a function of $A n m$ and $X n m$. The top-down approach is to find out an expression for $A n m$ as the function of $A n$ and $X n$.

$$
A n m=G(A n, X n)
$$

We expect that $F$ is a unique mapping, but $G$ can be a one-to-many mapping.

First I want to solve the aggregation problem from the national model to regional model. $H(X)$ denotes the diagonal matrix, in which the diagonal elements are elements of vector $(X)$.

Theorem 1. When $A n$ is fixed as follows, Multi-Sector-Multi-Region National model (2-1) and Multi-Regional model (2-3) are consistent.

$$
A n^{*}=\left(L R \cdot A n m \cdot H(X n m) \cdot L R^{\prime}\right)\left(L R \cdot H(X n m) \cdot L R^{\prime}\right)^{-1}
$$

(Proof) Using $X n=L R \cdot X n m, Y n=L R \cdot Y n m$, we show that $\left(I-A n^{*}\right) X n=Y n$

$$
\begin{aligned}
& \left(I-A n^{*}\right) \cdot X n=X n-A n^{*} \cdot X n=L R \cdot X n m-A n^{*} \cdot X n \\
= & L R \cdot X n m-\left(L R \cdot A n m \cdot H(X n m) \cdot L R^{\prime}\right)\left(L R \cdot H(X n m) \cdot L R^{\prime}\right)^{-1} L R \cdot X n m \\
= & L R \cdot X n m-(L R \cdot A n m \cdot H(X n m))\left(L R^{\prime}\right)\left(L R \cdot H(X n m) \cdot L R^{\prime}\right)^{-1} L R \cdot X n m \\
= & L R \cdot X n m-(L R \cdot A n m \cdot H(X n m)) J n m^{\prime} \\
= & L R \cdot X n m-L R \cdot A n m \cdot X n m=L R \cdot Y n m=Y n
\end{aligned}
$$


I used

$\left(L R^{\prime}\right)\left(L R \cdot H(X n m) \cdot L R^{\prime}\right)^{-1}(L R \cdot X n m)=J m^{\prime}$

So,

$$
\left(I-A n^{*}\right) \cdot X n=Y n
$$

We state the similar results for the consistency between other relations without the formal proofs.

Collorally 1 . When $A m$ is fixed as follows, the national model (2-1) and the sectoral model (2-2) are consistent.

$$
A m^{*}=\left(L S \cdot A n m \cdot H(X n m) \cdot L S^{\prime}\right)\left(L S \cdot H(X n m) \cdot L S^{\prime}\right)^{-1}
$$

Collorally 2. When $A a$ is fixed as follows, the regional model (2-3) and the macro model (24) are consistent.

$$
A a^{*}=\left(J n \cdot A n \cdot H(X n) \cdot J n^{\prime}\right)\left(J n \cdot H(X n) \cdot J n^{\prime}\right)^{-1}
$$

Collorally 3. When $A a$ is fixed as follows, the sectoral model (2-2) and the macro model (24) are consistent.

$$
A a^{* *}=\left(\mathrm{Jm} \cdot A m \cdot H(X m) \cdot J m^{\prime}\right)\left(\mathrm{Jm} \cdot H(X m) \cdot J m^{\prime}\right)^{-1}
$$

Finally we analyze the consistency between national and macro models.

Theorem 2. When the macro parameter $(A a)$ is fixed either as $A a^{*}$ or $A a^{* *}$, Multi-SectorMulti-Region National model and One-Sector-One-Region Macro model are consistent.

$$
\begin{aligned}
A a^{*}= & J n \cdot\left(L R \cdot A n m \cdot H(X n m) \cdot L R^{\prime}\right) \cdot\left(L R \cdot H(X n m) \cdot L R^{\prime}\right)^{-1} \cdot H(L R \cdot X n m) \\
& \cdot J n ' \cdot\left(J n \cdot H(J R \cdot X n m) \cdot J n^{\prime}\right)^{-1} \\
A a^{* *}= & J m \cdot\left(L S \cdot A n m \cdot H(X n m) \cdot L S^{\prime}\right) \cdot\left(L S \cdot H(X n m) \cdot L S^{\prime}\right)^{-1} \cdot H(L S \cdot X n m) \\
& \cdot J m^{\prime} \cdot\left(J m \cdot H(J S \cdot X n m) \cdot J m^{\prime}\right)^{-1}
\end{aligned}
$$

(Proof) Using $X a=J n m \cdot X n m$ and $Y a=J n m \cdot Y n m$, I show that $\left(1-a^{*}\right) \cdot X a=Y a$.

$$
\begin{aligned}
\left(1-a^{*}\right) \cdot X a= & \left(1-J n \cdot\left(L R \cdot A n m \cdot H(X n m) \cdot L R^{\prime}\right) \cdot\left(L R \cdot H(X n m) \cdot L R^{\prime}\right)^{-1} \cdot H(L R \cdot X n m)\right. \\
& \left.\cdot J n^{\prime} \cdot\left(J n \cdot H(J R \cdot X n m) \cdot J n^{\prime}\right)^{-1}\right) J n m \cdot X n m
\end{aligned}
$$

I use

$$
J n \cdot L R=J n m
$$

$$
\begin{gathered}
L R^{\prime} \cdot\left(L R \cdot H(X n m) \cdot L R^{\prime}\right)^{-1} \cdot H(L R \cdot X n m) \cdot J n^{\prime}=J n m^{\prime} \\
\left(J n \cdot H(J R \cdot X n m) \cdot J n^{\prime}\right)^{-1} \cdot J n m \cdot X n m=1
\end{gathered}
$$

Then

$$
\left(1-A a^{*}\right) \cdot J n m \cdot X n m=J n m \cdot Y n m
$$

\section{Numerical Example}

I take up a simple case of two-regions and two-sectors. The national model is specified as follows. $X(i, j)$ and $Y(i, j)$ denote the output and final demand of $i$-th sector and $j$-th region.

$$
\left[\begin{array}{cccc}
1-0.50 & -0.02 & -0.05 & -0.10 \\
-0.03 & 1-0.50 & -0.05 & -0.10 \\
-0.02 & -0.03 & 1-0.40 & -0.05 \\
-0.01 & -0.03 & -0.01 & 1-0.40
\end{array}\right]\left\{\begin{array}{l}
362.71(X N(1,1)) \\
465.84(X N(2,1)) \\
411.60(X N(1,2)) \\
514.60(X N(2,2))
\end{array}\right\}=\left\{\begin{array}{l}
100(Y N(1,1)) \\
150(Y N(2,1)) \\
200(Y N(1,2)) \\
250(Y N(2,2))
\end{array}\right\}
$$


The Leontief inverse matrices at national level and of other levels which are consistent with national model are as follows.

$$
\begin{aligned}
& \mathrm{MN}=\left[\begin{array}{llll}
2.0240 & 0.1180 & 0.2413 & 0.3771 \\
0.1401 & 2.0434 & 0.2460 & 0.3844 \\
0.0789 & 0.1164 & 1.7121 & 0.1752 \\
0.5390 & 0.1235 & 0.3016 & 1.7213
\end{array}\right]\left\{\begin{array}{l}
100 \\
150 \\
200 \\
250
\end{array}\right\}=\left\{\begin{array}{l}
362 \\
465 \\
411 \\
514
\end{array}\right\} \\
& \begin{array}{c}
M R=\left[\begin{array}{ll}
2.1654 & 0.6382 \\
0.1922 & 1.9514
\end{array}\right]\left\{\begin{array}{l}
250 \\
450
\end{array}\right\} \\
\text { (total) } 700 \quad\left\{\begin{array}{l}
828 \\
926
\end{array}\right\} \\
1754
\end{array} \\
& \mathrm{MI}=\left[\begin{array}{ll}
2.0203 & 0.4262 \\
0.4387 & 2.1512
\end{array}\right]\left\{\begin{array}{l}
300 \\
400
\end{array}\right\}=\left\{\begin{array}{l}
774 \\
980
\end{array}\right\} \\
& \text { (total) } 700 \quad 1754 \\
& \mathrm{MA}=2.526 \quad 700 \quad 1754
\end{aligned}
$$

Thus MN, MR, MI and MA are a set of mutually consistent inverse matrices each of which produces the same level of total output (1754) when multiplied by the corresponding adequate final demand vector.

\section{Prediction Error}

When the final demand increases by $\Delta Y n m$, the sectoral effect is calculated exactly as follows. $B m^{* *}$ and $B m^{*}$ denote the Leontief inverse matrices consistent with old and new final demand vectors.

$$
L S \cdot \Delta X n m=B m^{* *} \cdot L S \cdot(Y n m+\Delta Y n m)-B m^{*} \cdot L S \cdot Y n m
$$

The sectoral model predicts the effect as ;

$$
\Delta X m+=B m^{*} \cdot L S \cdot \Delta Y n m
$$

The error $(E m)$ between (4-2) and (4-1) is

$$
E m=\left(B m^{*}-B m^{* *}\right) \cdot L S \cdot(Y n m+\Delta Y n m)
$$

$B m^{*}$ and $B m^{* *}$ depend on the distribution vectors $\left(f m^{*}, f m^{* *}\right)$, and are free from the absolute level (JnmYnm and $J n m(Y n m+\Delta Y n m))$.

Theorem 3. Even if the sectoral coefficient matrix $(A m)$ is consistent with national coefficient matrix $(A n m)$, the sectoral effect of change in the national final demand $(\Delta Y n m)$ calculated by the sectoral model produces an error such as ;

$$
E m=\left(B m^{*}-B m^{* *}\right) \cdot L S \cdot(Y n m+\Delta Y n m)
$$

We assume that $B m$ is consistent with $A n m$. When the effect of an increment of sectoral final demand $(\Delta Y m)$ is calculated by $B m \cdot \Delta Y m,(4-4)$ gives the prediction error. $B m^{*}$ and $B m^{* *}$ depends on the distribution vectors $\left(\mathrm{fm}^{*}\right.$ and $\left.f \mathrm{~m}^{* *}\right)$ free from the absolute level. So the 
error does not vanish except the parallel increase $(\Delta Y n m=f n m \cdot \lambda, \lambda \neq 0)$.

Collorary 4. Even if the regional coefficient matrix $(A n)$ is consistent with national coefficient matrix $(\mathrm{Anm})$, the regional effect of change in the national final demand $(\Delta Y n m)$ calculated by the regional model produces an error such as;

$$
E n=\left(B n^{*}-B n^{* *}\right) \cdot L R \cdot(Y n m+\Delta Y n m)
$$

Let us calculate the error $(E N)$ by $(4-5)$ based on the numerical example cited before. We assume that the final demand for the 1st sector in region-1 increased by 100 . So $Y n m$ changed

$$
\operatorname{Ynm}(\text { new })=\left\{\begin{array}{l}
200 \\
150 \\
200 \\
250
\end{array}\right\}(\text { total }=800), Y n m(\text { old })=\left\{\begin{array}{l}
100 \\
150 \\
200 \\
250
\end{array}\right\}(\text { total }=700)
$$

Based on the national model, the new output is calculated exactly as 1984.49. The result by new consistent regional inverse matrix $\left(B m^{* *}\right)$ is :

$$
\begin{gathered}
B m^{* *} L R(Y n m+\Delta Y n m)=\left[\begin{array}{ll}
2.16589 & 0.637643 \\
0.179682 & 1.948010
\end{array}\right]\left\{\begin{array}{l}
350 \\
450
\end{array}\right\}=\left\{\begin{array}{r}
1045.08 \\
945.40
\end{array}\right\} \\
B m^{*}-B m^{* *}=\left[\begin{array}{cc}
0.00049 & (-) 0.000557 \\
(-) 0.12512 & (-) 0.003390
\end{array}\right]\left\{\begin{array}{l}
350 \\
450
\end{array}\right\}=\left\{\begin{array}{l}
(-) 0.0792 \\
(-) 5.9047
\end{array}\right\} \\
(\text { total) } 800 \quad(-) 5.9839
\end{gathered}
$$

The use of old matrix projected the total output by 1990.48 , and created the error by (-)5.984. The same error is calculated by (4-8). This error is solely due to the changes in technical coefficient matrix or Leontief inverse matrix based on the distributional change of final demand vector. The change in final demand amounted to 14.28 per cent $(100 / 700)$. Then the relative error was 0.3 per cent $(5.98 / 1984.49)$. But the error concentrated to the second sector, and her output was projected as 945 instead of true value of 939 . So the relative error (6/939) amounted to 0.6 per cent. This is far big to be neglected. Perhaps the input coefficient from sector 2 to sector 1 (a21) was influenced mostly, and produced a big error for sector 2. If we calculate the marginal ratio (error divided by the increment of output, 5.984/ $230.49)$, the relative error ratio amounts to 2.59 per cent. So the misspecification error becomes quite big.

\section{Top-Down Approach}

Now we turn to top-down approach, in which the coefficient matrix of lower level of aggregation must be inferred by the information at higher level. For example, when there is a set of consistent matrices at a certain point of time, and the matrix of higher level is extrapolated somehow, we want to infer the matrices of lower levels. Naturally there are 
some degrees of freedom to manipulate, so we are interested in their efficient utilization.

We take up a simplest case. First we assume that at the initial period, there exist a set of consistent matrices and vectors (with ${ }^{*}$ symbols) :

$$
\begin{gathered}
\left(1-A a^{*}\right) \cdot X a^{*}=Y a^{*} \\
\left(U m-A m^{*}\right) \cdot X m^{*}=Y m^{*}
\end{gathered}
$$

Then at another period, the following macro model is projected.

$$
(1-A a) \cdot X a=Y a \quad(A a, X a, Y a \text { are given })
$$

Then the question is what are the appropriate coefficients of the regional model :

$$
(U m-A m) \cdot X m=Y m
$$

From (5-1) to (5-4),

$$
\begin{gathered}
J m^{\prime} \cdot X m=X a \\
J m^{\prime} \cdot Y m=Y a \\
J m^{\prime} \cdot A m \cdot X m=A a \cdot X a
\end{gathered}
$$

In (5-4), there are $\left(2 m+m^{2}\right)$ variables : $X m(m), Y m(m)$ and $A m\left(m^{2}\right)$. There are $(m+$ $3)$ restraints : $(5-2)(m),(5-3)(1),(5-4)(1),(5-6)(1)$. The degree of freedom $(D F)$ increases as $\mathrm{m}$ increases from 2 .

$\begin{array}{lccccc} & \text { Variables }\left(2 m+m^{2}\right) & \text { Restraints }(m+3) & \text { Degree of Freedom } \\ m=2 & 8 & - & 5 & = & 3 \\ m=3 & 15 & - & 6 & = & 9 \\ m=4 & 24 & - & 7 & = & 17\end{array}$

First assume $m$ as 2, and write the elements of $A m$ as aij

$$
A 2=\left[\begin{array}{ll}
a 11 & a 12 \\
a 21 & a 22
\end{array}\right], \quad X 2=\left\{\begin{array}{l}
x 1 \\
x 2
\end{array}\right\}, \quad Y 2=\left\{\begin{array}{l}
y 1 \\
y 2
\end{array}\right\}
$$

Then (5-4) to (5-7) can be written as :

$$
\begin{gathered}
\left(\left[\begin{array}{ll}
1 & 0 \\
0 & 1
\end{array}\right]-\left[\begin{array}{ll}
a 11 & a 12 \\
a 21 & a 22
\end{array}\right]\right) \cdot\left\{\begin{array}{l}
x 1 \\
x 2
\end{array}\right\}=\left\{\begin{array}{l}
y 1 \\
y 2
\end{array}\right\} \\
x 1+x 2=X a \\
y 1+y 2=Y a \\
(1,1) \cdot\left[\begin{array}{ll}
a 11 & a 12 \\
a 21 & a 22
\end{array}\right]\left\{\begin{array}{l}
x 1 \\
x 2
\end{array}\right\}=A a \cdot X a
\end{gathered}
$$

The degree of freedom is three. So we cannot fix all aij completely. Let us imagine that aij matrix changes in RAS fashion :

$$
\left[\begin{array}{cc}
r 1 & 0 \\
0 & r 2
\end{array}\right] \cdot\left[\begin{array}{cc}
a 11^{*} & a 12^{*} \\
a 21^{*} & a 22^{*}
\end{array}\right] \cdot\left[\begin{array}{cc}
s 1 & 0 \\
0 & s 2
\end{array}\right]=\left[\begin{array}{cc}
a 11 & a 12 \\
a 21 & a 22
\end{array}\right]
$$

Then we can fix anyone out of 4 variables $(r 1, r 2, s 1, s 2)$. So one of plausible way is:

(1) Assume $r 1, r 2, s 1$ and calculate $s 2, x 1, x 2, y 1, y 2$. These values satisfy all the 
constraints (5-8) (5-9) (5-10) (5-11).

(2) Repeat the procedures by assuming different sets of $(r 1, r 2, s 1)$.

Another way can be :

(1) Set there of four $(r 1, r 2, s 1, s 2)$ as unity, and calculate the one.

(2) Repeat the procedure four times, and let the solutions as $r 1+, r 2+, s 1+$ and $s 2+$.

(3) Make $\lambda(r 1+, r 2+, s 1+, s 2+) / 4$ as tentative solution $(\lambda=1)$. And finally fix the value of $\lambda$, which satisfies all the constraints.

When DF varies, we can set up a similar procedure.

\section{Comparison of Optimum Solutions-Technological Difference and Hysteresis}

Now we engage in a consistency approach. We assume that each of the different models at different levels of aggregation reflects a special aspect of the reality. Then how we can reconcile between different models?

We assume that the economy faces with capital and labor constraints, and compare two national model $(N M)$ versus sectoral models $(S M)$. We classify the cases as for the interregional mobility and the technology :

(A1) assumption of free mobility : $\mathrm{knm}(\mathrm{lnm})$ are capital (labor) coefficients mn-dimension vectors, $\mathrm{km}$ and $\mathrm{lm}$ are labor coefficients m-dimension vectors, and $C$ and $E$ denote endowment of the total national capital and labor.

$$
k n m \cdot X n m \leqq C(N M), \quad l n m \cdot X n m \leqq E(N M)
$$

(A2) assumption of no mobility ( $\mathrm{Cm}$ and $\mathrm{Em}$ are $\mathrm{m}$ dimension column vectors, and $K n m^{*}$ and $\mathrm{Lnm}^{*}$ denote the matrices formed by collocating vectors $\mathrm{knm}$ and $\mathrm{lnm}$ in suitable manners. $\mathrm{km}^{*}$ and $l \mathrm{~m}^{*}$ are diagonal matrices formed by $\mathrm{km}$ and $l m$ vectors) :

$$
\begin{array}{cl}
K n m^{*} \cdot X n m \leqq C m(N M), & L n m^{*} \cdot X n m \leqq E m(N M) \\
k m^{*} \cdot X m \leqq C m(S M), & l m^{*} \cdot X m \leqq E m(S M)
\end{array}
$$

The lack of mobility comes either from the region-specific capital or the fact that the capital is not region-specific but the movement of capital takes time (hysteresis).

(B1) Common technologies :

$$
\begin{gathered}
A n m=\left[\begin{array}{cccc}
A m, & 0, & \cdots & 0, \\
0, & A m, & \cdots & 0 \\
0, & 0, & \cdots & 0, \\
0, & 0, & \cdots & A m
\end{array}\right] \\
K n m=(K m, \cdots, K m) \\
L n m=(L m, \cdots, L m)
\end{gathered}
$$


(B2) Different technology : each region has different coefficients. So input coefficients $(A i j)$, capital and labor coefficients $(k j, l j)$ are different between regions.

Let us compare two models :

Model-N. National (Sector-Region) Model :

$$
\begin{gathered}
\text { Max Pnm' } \cdot Y n m \\
(U n m-A n m) \cdot X n m \geqq Y n m \\
k n m \cdot X n m \leqq C \\
\operatorname{lnm} \cdot X n m \leqq E \\
X n m \geqq 0
\end{gathered}
$$

Model-S. Sectoral Model :

$$
\begin{gathered}
\operatorname{Max} P m^{\prime} \cdot Y m \\
(U m-A m) \cdot X m \geqq Y m \\
k m \cdot X m \leqq K m \\
l m \cdot X m \leqq L m \\
X m \geqq 0
\end{gathered}
$$

We postulate that $L S \cdot P m=P n m$, that is, the evaluation of a product for final use is common in every region. Then we ask which is bigger among $P n m^{\prime} \cdot Y n m$ and $P m^{\prime} \cdot Y m$. Naturally the conclusion depends on the technical conditions (the size of coefficients $(A, k, l)$, and the availability of resources in each region. These separation creates four cases : Case ( $A 1-B 1),(A 1-B 2),(A 2-B 1)$ and $(A 2-B 2)$. The case $(A 1-B 1)$ is not interesting, because $P n m^{\prime} \cdot Y n m$ automatically equals to $P m^{\prime} \cdot Y m$. For other combinations, naturally the answer depends on several crucial conditions.

In case of same technology, (6-8) can be written as

So,

$$
L S \cdot(U n m-A n m) \cdot X n m \geqq L S \cdot Y n m
$$

Also $(6-9)$ and $(6-10)$ can be written as ;

$$
(U m-A m) \cdot L S \cdot X n m \geqq L S \cdot Y n m
$$

$$
\begin{aligned}
& K m \cdot L S \cdot X n m \leqq C \\
& L m \cdot L S \cdot X n m \leqq E
\end{aligned}
$$

There are two cases in which the solutions of two models coincide : (1) same technology and free mobility, and (2) $A m, K m$ and $L m$ are consistent with the national coefficients, Anm, Knm and Lnm, defined as (2-26) and (6-20) and (6-21).

$$
\begin{aligned}
& K m=\left(L S \cdot K n m \cdot H(X n m) \cdot L S^{\prime}\right)\left(L S \cdot H(X n m) \cdot L S^{\prime}\right)^{-1} \\
& L m=\left(L S \cdot L n m \cdot H(X n m) \cdot L S^{\prime}\right)\left(L S \cdot H(X n m) \cdot L S^{\prime}\right)^{-1}
\end{aligned}
$$

Then we assume the different technology, and write the optimum solution of the national model with free mobility, (6-7)-(6-11), as $X n m^{*}$. If the sectoral coefficients are consistent with $X n m^{*}$ and national coefficients, the solution of sectoral model $\left(X m^{*}\right)$ coincides with $J S$ $\cdot X n m^{*}$. If not, $\mathrm{Jm} \cdot \mathrm{Ym}\left(=\mathrm{Jm} \cdot \mathrm{Bm} \cdot \mathrm{Xm^{* }}\right)$ is smaller than $\mathrm{Jnm} \cdot \mathrm{Bnm} \cdot \mathrm{Xnm} \mathrm{m}^{*}$. When (6-1) is 


\section{Fukuchi}

replaced with (6-2), in general, $X n m^{*}$ is not feasible. Let us write the optimum solution as $X n m+$. Then, in general,

$$
J n m \cdot B n m \cdot X n m^{*} \geqq J n m \cdot B n m \cdot X n m+
$$

Therefore, the different technologies among regions makes the value of target function higher, while the lack of mobility of resources makes the value lower. These two factors make the comparison of values of target functions of national model with same regional technology and free mobility and of national model with different regional technology and hysteresis makes ambiguous.

In reality, the sectoral technology is different among regions. The differences may be due to the different product mix, but at least partly due to different technology based on scale merit and regional specific factors. Also the mobility of resources is not perfect. So the conclusion is not decisive.

\section{Summary and Conclusions}

In some countries, mn-dimension national table ( $\mathrm{m}$-sector and $\mathrm{n}$-regions), $\mathrm{n}$-dimension regional table, $\mathrm{m}$-dimension sectoral table, and finally the simplest (1-sector and 1-region) multiplier equation are available. Then which table has the highest statistical reliability ? Which table is most relevant to reality ? By asking these questions, we face with the aggregation problems between tables of different levels of aggregation. In this paper, I followed the traditional three approaches to this problem.

Based on the Bottom-Up Approach, I showed that the aggregated tables could project the same multiplier effects when the coefficients are defined in consistent ways. I also analyzed the prediction errors when non-consistent table is used to projections. Top-Down Approach suggested a procedure to derive the disaggregated table by a programming type calculation. In the consistency approach, I took up two factors : different technologies and lack of resource mobility (hysteresis), and argued that the maximized output can be bigger or smaller in an aggregated table.

Basically this paper is a methodological essay, which still I believe useful to discuss many fresh issues although additional issues might come out in case of actual manipulations.

\section{References}

Hatanaka, M., 1952, Note on consolidation within a Leontief system, Econometrica, vol. 27, 301-303.

Hewings, G. J. D., and R. C. Jensen, 1986, Regional, interregional and multiregional input - output analysis, in P. Nijkamp (ed), Handbook of Regional and Urban Economics, vol. 1, 295-355, Amsterdam, NorthHolland.

McManus, M., 1956, On Hatanaka's note on consolidation, Econometrica, vol. 24, 482-487. 


\section{Aggregation Problem in Multi-Regional Input-Output Models}

Nijkamp, P., P. Rietveld and F. Snickers, 1986, Regional and multiregional economic models : A survey, in

P. Nijkamp (ed), Handbook of Regional and Urban Economics, vol. 1, 257-294, Amsterdam, NorthHolland.

Theil, H., 1954, Linear Aggregation of Economic Relations, Amsterdam, North-Holland, pp. 1-205. 